\title{
Surgical Management of Simple Liver Cysts: A 10-Year Single-Center Experience
}

\author{
Oludolapo Sotade ${ }^{\mathrm{a}, \mathrm{b}}$, Omar Mouline ${ }^{\mathrm{a}}$, Carlo Pulitano ${ }^{\mathrm{a}}$, Michael Crawford ${ }^{\mathrm{a}}$, David Joseph ${ }^{\mathrm{a}}$, James Gallagher ${ }^{\mathrm{a}}$, \\ Charbel Sandroussia
}

\begin{abstract}
Background: Due to the limited data comparing treatment outcomes of simple liver cysts (SLCs), there is, at present, no consensus on the optimum surgical treatment method for symptomatic SLCs. The objective of this paper was to review the outcomes for surgically managed SLCs carried out at our institution.
\end{abstract}

Methods: A database search was performed to identify all patients who, between January 2003 and December 2012, underwent surgical intervention with a preoperative diagnosis of symptomatic SLCs at our institution. Retrospective analysis of patient demographics, symptoms, cyst characteristics including number, size, location and imaging features, operative management, postoperative morbidity and mortality, length of hospital stay, final histopathological diagnosis and long-term outcomes were performed.

Results: Between January 2003 and December 2012, 28 patients underwent surgical intervention for symptomatic SLCs at our institution. Twenty-four were female (85.7\%) and four were male (14.3\%), with a mean age of 58 years. Laparoscopic fenestration was performed in 11 patients $(39.3 \%)$, open fenestration in four $(14.3 \%)$, laparoscopic resection in six (21.4\%) and open resection in seven (25\%). The mean cyst diameter was $8.97 \pm 5.11 \mathrm{~cm}$. Operative mortality was $3.6 \%(\mathrm{n}=$ 1). Complications developed in two patients (7.2\%), including pleural effusion $(n=1)$ and perihepatic abscess $(n=1)$. The final histopathology demonstrated an SLC in 23 patients $(82.1 \%)$, cystadenoma in four $(14.3 \%)$ and cystadenocarcinoma in one patient (3.6\%). Length of hospital stay was $2.38 \pm 2.06$ days for the laparoscopic group and $7 \pm 3.12$ days for the open surgery group. Two patients (7.14\%) experienced asymptomatic recurrence of cyst. The mean length of followup was 25.1 months (range 12 - 104.1) and the overall survival rate at 1 year was $96.4 \%$.

Conclusion: Laparoscopic fenestration is a safe and effective treat-

Manuscript accepted for publication March 30, 2015

aRoyal Prince Alfred Hospital, Upper Gastrointestinal and Hepatobiliary Surgery, Sydney, New South Wales, Australia

bCorresponding Author: Oludolapo Sotade, Royal Prince Alfred Hospital, Missenden Road Camperdown, NSW 2050, Australia.

Email: d.sotades@gmail.com

doi: http://dx.doi.org/10.14740/jcs263w ment for symptomatic SLCs. Open fenestration should be considered for large cysts, cysts presenting difficult laparoscopic access, and cysts with atypical features. Atypical features should prompt intraoperative frozen section and, if necessary, liver resection.

Keywords: Simple liver cyst; Fenestration; Resection

\section{Introduction}

Liver cysts may arise from the biliary tree or from hepatic tissue. Liver cysts include non-parasitic simple liver cysts (SLCs), multiple cysts that occupy less than $50 \%$ of the liver volume, polycystic disease that occupies more than $50 \%$ of the liver volume and acquired cysts, which may be the result of parasitic, bacterial, or amoebic infection. Proliferative lesions or tumors may also arise, including cystadenocarcinomas, or benign like cystadenomas [1].

The incidence of SLC is $2.5-5 \%$ [2-5]. The vast majority of SLC are asymptomatic and the diagnosis of SLC is generally an incidental finding on abdominal imaging or abdominal surgery for unrelated pathology. There is a female preponderance for symptomatic SLC, with a ratio of 9:1 [4, 6-8]. The prevalence of SLC is higher in females, and is markedly increased between the ages of 40 and 60 years, as reported by Caremani et al [9]. The $5-10 \%$ of SLC that present with symptoms do so because of mechanical compression, rupture, hemorrhage into the cyst, or infection [10].

SLCs are usually thin-walled without mural nodules or papillary projections. Septa are absent, giving rise to a unilocular clear cyst. Asymptomatic SLC is managed conservatively. Once symptoms or complications develop, surgical options may include fenestration and resection of the cyst using a laparoscopic or open approach. Other alternatives include percutaneous aspiration and alcohol sclerotherapy but these are less effective and have a high recurrence rate of $100 \%$ and $75 \%$ respectively $[1,2,4,6]$.

Due to the limited number of patients and follow-up SNP data available, comparisons of the various operative techniques have been sub-optimal and the optimum surgical management of SLC has not been clearly established [1]. The literature suggests recurrence rates of $8-25 \%$ after laparoscopic fenestration and $13 \%$ after open fenestration. However, liver resection is 
not reported to have associated cyst recurrence [3, 5, 11-15]. This study evaluates our experience in a single-center over 10 years to determine the optimum operative treatment for SLC.

\section{Methods}

Institutional ethics approval was obtained for this study. Twenty-eight patients with a preoperative diagnosis of SLC who underwent surgical treatment at our institution between January 2003 and December 2012 were identified by cross-referencing our surgical and pathology databases. Patient demographics, perioperative parameters, final histopathology, and long-term outcomes were reviewed. Patient morbidity was graded using Dindo-Clavien's classification system [16].

Preoperative blood CA 19-9 and CEA was obtained in seven patients. Cyst fluid aspiration was performed in 11 patients. The cyst fluid was assayed for CA 19-9 in only one patient. Preoperative computer tomography (CT) of the lesion was carried out in all but one patient. Preoperative MRI was performed in six patients. All patients had preoperative ultrasonography examinations. These imaging tests were designed to test parameters including the presence of multilocularity, septa, mural nodules, papillary projections or any other solid component in the tumor, which are classically markers of nonsimple cysts. Other criteria were the presence of calcification, increased vascularity, and cyst wall enhancement following wall contrast injection.

Percutaneous drainage was attempted two times in one patient, and one other percutaneous drainage procedure was performed. The types of surgery were classified by the approach as laparoscopic, laparoscopic converted to open surgery, and open surgery. The surgical procedures used in each case were recorded from the operative notes. The procedures included fenestration, segmental resection, bisegmentectomy, and hemi-hepatectomy. Frozen section was performed in two cases. Operative mortality was defined as death within 90 days of the surgical procedure.

Length of follow-up was from the date of the procedure to the date of the last imaging result, or date of last clinical review, within our institution or affiliates. Follow-up was performed by clinical examination and laboratory studies. Additional investigations including ultrasound (US) of the abdomen was performed depending on the clinical indication. Patients were contacted by telephone and asked to corroborate the most recent cyst recurrence status.

Cyst recurrence was defined as symptomatic recurrence or radiological/asymptomatic recurrence of the same type of cyst in the same spot or another location.

\section{Results}

Between January 2003 and December 2012, 28 patients underwent surgical intervention for a preoperative diagnosis of SLC at our institution. The mean age at surgery was 58.2 (SD \pm 13.7 , range $18-74$ years). There were 24 female and four male patients. Of the 28 patients, four patients $(14.3 \%)$ were asymptomatic on presentation. Twenty-four patients presented with initial symptoms, of which abdominal pain (71.4\%), abdominal fullness $(17.9 \%)$, nausea and vomiting $(14.3 \%)$ were most typical. Other symptoms included early satiety, back pain, dyspnea and cough [1].

Preoperative CA 19-9 and CEA values from seven patients ranged between less than $2-105 \mathrm{ng} / \mathrm{dL}$ and $0.6-9 \mathrm{IU} / \mathrm{mL}$. There was no clear cut-off between an SLC, cystadenoma, or cystadenocarcinoma based on these measurements.

Preoperative abdominal US testing and CT assessment were performed on 28 and 27 patients respectively. These revealed the presence of multilocular cysts in $14(51.85 \%)$. Septa were present in $15(55 \%)$ of the observed cysts. Accordingly, these were not useful in differentiating between SLC and other cystic lesions. The presence of a mural nodule, papillary projection or other solid component was detected in six out of 27 cysts $(22.22 \%)$. This included the single cystadenocarcinoma and two of the four cystadenomas in the study. Calcification was present in four of the cases. Hypervascularity was a phenomenon observed in eight (29.6\%). Contrast injection was followed by increased cyst wall visualization in all of the cystadenomas, but only one SLC. However, the malignant lesion did not show enhancement. Examination of the biliary duct showed proximal dilatation in six patients, but this was nondiscriminative.

Magnetic resonance imaging (MRI) was performed on six patients $(21.4 \%)$, three of whom were subsequently proved to have cystadenomas on final histopathology. The one patient who was found to have a cystadenocarcinoma had no MRI examination before surgery. One patient had aspirated cyst fluid tested for CA 19-9 (with a serum CA 19-9 of $93 \mathrm{ng} / \mathrm{dL}$ ) which was found to be $156 \mathrm{U} / \mathrm{mL}$.

One patient had an incidental $10.9 \mathrm{~cm}$ cyst and was offered treatment due to the significant potential for cyst complications (the final histopathology revealed it to be a cystadenoma). Laparoscopic surgery was carried out in 17 cases, and the remaining 11 patients had open surgery. Types of operative procedures included six laparoscopic resections and seven open resections (one hemi-hepatectomy, five segmentectomies, and one bi-segmentectomy). Fenestration or unroofing was performed in 15 patients (48\%), 11 laparoscopic and four open fenestration procedures were performed.

Mean operative time was 91.8 min (range 10 - $264 \mathrm{~min}$ ). Mean operative time was $27.5 \pm 15.41 \mathrm{~min}$ for laparoscopic fenestration, $84.25 \pm 14.34 \mathrm{~min}$ for open fenestration, 99.33 $\pm 48.92 \mathrm{~min}$ for laparoscopic resection and $184.5 \pm 86.04 \mathrm{~min}$ for open resection.

Cyst size was recorded as the greatest cystic diameter. The largest lesion diameter ranged from 2 to $22 \mathrm{~cm}$. Mean cyst size was $8.97 \pm 5.11 \mathrm{~cm}$ for all cysts and $9.48 \pm 5.15 \mathrm{~cm}$ for symptomatic cysts. The number of cystic lesions ranged from one to five. Twelve patients had a primary cyst in the right lobe (43\%) and nine in the left lobe (32\%); seven patients $(25 \%)$ had bilateral cysts. Twenty patients $(71.4 \%)$ had one dominant cyst and eight patients $(28.6 \%)$ had several other asymptomatic cysts detected on imaging. Overall, the mean postoperative length of hospital stay was 4 days $(2.38 \pm 2.06$ days for laparoscopic procedures and $7 \pm 3.12$ days for open procedures). Frozen section was performed in two cases, one of which was subse- 
quently found to be a cystadenoma and the other a cystadenocarcinoma. The final histopathology was SLC in 23 patients $(82.1 \%)$, cystadenoma in four patients $(14.3 \%)$, and cystadenocarcinoma $(3.6 \%)$. The patient with cystadenocarcinoma subsequently underwent open resection.

One patient $(3.6 \%)$ died of cardiac and respiratory failure on the sixth postoperative day following open left hemi-hepatectomy. An additional two patients (7.2\%) developed complications. One patient developed a pleural effusion (grade I-d) following open right hepatectomy and thus no further intervention was required. The second patient developed a perihepatic abscess and a pleural effusion (grade IIIa-d) following bisegmentectomy, requiring antibiotics and CT-guided drainage.

Mean length of follow-up was 25.1 months (range 12 104.1). There were no symptomatic recurrences. Two patients (7.14\%) were observed to have asymptomatic recurrence. The asymptomatic recurrence of SLC occurred during follow-up at 2.5 and 17.4 months for open fenestration and laparoscopic fenestration procedures respectively. In these cases, both recurrences were discovered during follow-up. Neither required treatment. This is consistent with existing studies, which show that recurrences are rarely symptomatic [10].

\section{Discussion}

Adequate differentiation of the various cystic liver disorders is necessary for directing a suitable management plan. Polycystic liver disease (PCLD) is a congenital liver disease and is diagnosed when $>20$ liver cysts are present $[1,17,18]$.

A number of identifying characteristics found on US and CT investigation make it possible to distinguish SLC from most other lesions [19]. However, it can be challenging to differentiate SLC from cystadenoma and cystadenocarcinoma on imaging alone, as they can exhibit joint radiographic features $[3,4,7,12,19]$. In our study too, imaging failed to differentiate adequately between simple and complex cysts or between benign and malignant cystic lesions.

Among our patients, preoperative diagnosis of SLC was based on US and CT findings. Kim et al compared the CT features of cystadenoma and cystadenocarcinoma to CT features of atypical SLC that showed septation and calcification [19]. It was observed that a left lobe cyst, upstream biliary dilatation, number of cysts $<3$ and a positive perilesional transhepatic attenuation difference (THAD) were predictors of malignancy $[19,20]$. The atypical appearance of SLC on cross-sectional images is also attributable to previous complications such as cyst rupture, hemorrhage or infection $[4,10]$.

The high rate of hepatic resection in our series may be attributed to the difficulty in ruling out complex and malignant cystic lesions by imaging and serum studies. In most series, cystadenocarcinomas and cystadenomas represent less than $5 \%$ of cystic liver lesions $[1,19]$. However, in this center, they accounted for $17.8 \%$ of the total number of lesions thought to be SLC. A cystadenocarcinoma is a malignant neoplasm and therefore resection is the definitive management. Due to their potential for malignant transformation, formal resection is also advised for cystadenomas [1, 4, 21, 22].
Four of our patients were asymptomatic leading up to surgery. Two of these patients had a history of colorectal cancer (CRC). They were offered surgery because of the non-specificity of current imaging, tumor marker technology, and risk of missing a possible metachronous hepatic metastasis.

Laparoscopic fenestration is a procedure in which the roof of the cyst is removed as much as possible, draining the cyst and preventing re-accumulation of the cyst fluid. In addition, partial excision of the liver may be combined with this procedure if the cystic part of the liver is practically devoid of functional tissue. Laparoscopic fenestration provides several advantages over an open technique, including lower mortality and morbidity, reduced operative time, and shorter length of stay. Our results are consistent with the literature in this respect. Reported cyst recurrence rates following laparoscopic fenestration of SLC range between $8 \%$ and $25 \%$ [3, 5, 11-15]. Recurrence has been linked to incomplete unroofing, laparoscopic unroofing of a recurrent cyst which almost always has adhesions making complete deroofing difficult, deep location of the cyst and posterior segment cysts [1]. We observed one $(9 \%)$ asymptomatic recurrence at 17.4 months following laparoscopic fenestration. Predictors of recurrence include incomplete fenestration, previous operative treatment, deep-seated cysts, and cysts located within the posterior liver segments [1, $13]$.

Liver resection is undoubtedly a highly effective treatment alternative for the management for the majority of cystic disorders [1]. It is associated with very low recurrence rates. However, it carries a significantly greater risk for complications [11]. In our series, we performed 10 resections for SLC (four laparoscopic and six open), three resections for cystadenoma (two laparoscopic and one open) and one (open) resection for cystadenocarcinoma. The large number of resections reflects the prevalence of cyst resection as a safe and effective practice with no risk of recurrence, established over decades of experience in a long-running center like ours.

Within the laparoscopic group, there were no complications or cyst recurrence. There were no reported recurrences after open resection likewise. However, three patients did have postoperative complications after resection, which account for all such within this study. Open resection is best reserved for patients who have lesions suspicious of proliferative or malignant lesions [11].

Laparoscopic fenestration is a safe and effective treatment modality for symptomatic SLCs. It is associated with shorter operative times, less blood loss, lower morbidity, and shorter length of hospital stay, but carries a higher recurrence rate relative to the other surgical treatment options. Omentoplasty and the use of the argon laser to coagulate the cyst wall after fenestration are reported to minimize recurrences with this technique [11]. Open fenestration should be considered for large cysts, cysts presenting difficult laparoscopic access and cysts with atypical features. Atypical features should also prompt intraoperative frozen section and, if necessary, liver resection.

This study has limitations such as a small size, relatively short period of follow-up, failure to diagnose SLC in contrast with other proliferative cystic lesions, and varying use of imaging and serum studies between patients. Its strength lies in 
the inclusion of radiological recurrence and the verification of recurrence status at the time of reporting.

In order to determine the optimum operative management of SLC, a randomized controlled trial utilizing both resection and fenestration in a large sample of patients presenting with this condition is required to evaluate the safety and efficacy of laparoscopic and open methods of resection and fenestration.

\section{Conflicts of Interest}

None declared.

\section{References}

1. Garcea G, Rajesh A, Dennison AR. Surgical management of cystic lesions in the liver. ANZ J Surg. 2013;83(78):E3-E20.

2. Garcea G, Pattenden CJ, Stephenson J, Dennison AR, Berry DP. Nine-year single-center experience with nonparastic liver cysts: diagnosis and management. Dig Dis Sci. 2007;52(1):185-191.

3. Martin IJ, McKinley AJ, Currie EJ, Holmes P, Garden OJ. Tailoring the management of nonparasitic liver cysts. Ann Surg. 1998;228(2):167-172.

4. Brotzakis P, Mitellas T, Karaliotas CC. Non-parasitic diseases of the liver and intrahepatic biliary tree. Liver and biliary tract surgery: Springer; 2006. p. 515-526.

5. Tocchi A, Mazzoni G, Costa G, Cassini D, Bettelli E, Agostini N, Miccini M. Symptomatic nonparasitic hepatic cysts: options for and results of surgical management. Arch Surg. 2002;137(2):154-158.

6. Salemis NS, Georgoulis E, Gourgiotis S, Tsohataridis E. Spontaneous rupture of a giant non parasitic hepatic cyst presenting as an acute surgical abdomen. Ann Hepatol. 2007;6(3):190-193.

7. Moorthy K, Mihssin N, Houghton PW. The management of simple hepatic cysts: sclerotherapy or laparoscopic fenestration. Ann R Coll Surg Engl. 2001;83(6):409-414.

8. Benhamou J, Menu Y. Non-parasitic cystic diseases of the liver and intrahepatic biliary tree. Surgery of the liver and biliary tract, 1st ed. Great Britain, UK: Churchill Livingstone. 1988:1013-1024.

9. Caremani M, Vincenti A, Benci A, Sassoli S, Tacconi D. Ecographic epidemiology of non-parasitic hepatic cysts. J Clin Ultrasound. 1993;21(2):115-118.

10. Fabiani P, Iannelli A, Chevallier P, Benchimol D, Bour- geon A, Gugenheim J. Long-term outcome after laparoscopic fenestration of symptomatic simple cysts of the liver. Br J Surg. 2005;92(5):596-597.

11. Tan YM, Chung A, Mack P, Chow P, Khin LW, Ooi LL. Role of fenestration and resection for symptomatic solitary liver cysts. ANZ J Surg. 2005;75(7):577-580.

12. Ruiz-Tovar J, Lopez-Buenadicha A, Moreno-Caparros A, Vazquez-Garza JN. Surgical management of simple liver cysts. Cir Cir. 2012;80(1):52-55.

13. Gigot JF, Legrand M, Hubens G, de Canniere L, Wibin E, Deweer F, Druart ML, et al. Laparoscopic treatment of nonparasitic liver cysts: adequate selection of patients and surgical technique. World J Surg. 1996;20(5):556561.

14. Regev A, Reddy KR, Berho M, Sleeman D, Levi JU, Livingstone AS, Levi D, et al. Large cystic lesions of the liver in adults: a 15-year experience in a tertiary center. $\mathrm{J}$ Am Coll Surg. 2001;193(1):36-45.

15. Koperna T, Vogl S, Satzinger U, Schulz F. Nonparasitic cysts of the liver: results and options of surgical treatment. World J Surg. 1997;21(8):850-854; discussion 854855.

16. Dindo D, Demartines N, Clavien PA. Classification of surgical complications: a new proposal with evaluation in a cohort of 6336 patients and results of a survey. Ann Surg. 2004;240(2):205-213.

17. Gevers TJ, Drenth JP. Diagnosis and management of polycystic liver disease. Nat Rev Gastroenterol Hepatol. 2013;10(2):101-108.

18. Fedeles SV, Gallagher AR, Somlo S. Polycystin-1: a master regulator of intersecting cystic pathways. Trends Mol Med. 2014;20(5):251-260.

19. Kim JY, Kim SH, Eun HW, Lee MW, Lee JY, Han JK, Choi BI. Differentiation between biliary cystic neoplasms and simple cysts of the liver: accuracy of CT. AJR Am J Roentgenol. 2010;195(5):1142-1148.

20. Kim HJ, Kim AY, Kim TK, Byun JH, Won HJ, Kim KW, Shin YM, et al. Transient hepatic attenuation differences in focal hepatic lesions: dynamic CT features. AJR Am J Roentgenol. 2005;184(1):83-90.

21. Fiamingo P, Tedeschi U, Veroux M, Cillo U, Brolese A, Da Rold A, Madia C, et al. Laparoscopic treatment of simple hepatic cysts and polycystic liver disease. Surg Endosc. 2003;17(4):623-626.

22. Delis SG, Touloumis Z, Bakoyiannis A, Tassopoulos N, Paraskeva K, Athanassiou K, Safioleas M, et al. Intrahepatic biliary cystadenoma: a need for radical resection. Eur J Gastroenterol Hepatol. 2008;20(1):10-14. 